\title{
Joule heating in high magnetic field pulsars
}

\author{
V. Urpin ${ }^{1,2}$ and D. Konenkov ${ }^{2}$ \\ 1 Departament de Física Aplicada, Universitat d'Alacant, Ap. Correus 99, 03080 Alacant, Spain \\ e-mail: Vadim.Urpin@uv.es \\ 2 A.F.Ioffe Institute of Physics and Technology and Isaac Newton Institute of Chile, Branch in St. Petersburg, 194021 St. Petersburg, \\ Russia
}

Received 27 August 2007 / Accepted 15 February 2008

\begin{abstract}
Aims. We study the efficiency of Joule heating in the crustal layers of young neutron stars.

Methods. The induction equation is solved numerically for a realistic crust model with a conductivity that depends on the density and temperature.

Results. We show that dissipation of the magnetic field is highly inhomogeneous in the crust with much faster dissipation in relatively low density layers. In young neutron stars, the rate of Joule heating in the crust can exceed the standard luminosity of non-magnetic stars and can even be comparable to the luminosity of magnetars.

Conclusions. The results of calculations are compared with the available observational data. We argued that the crustal field model can well account for the data on the surface temperature and magnetic field of young neutron stars.
\end{abstract}

Key words. magnetohydrodynamics (MHD) - stars: neutron - stars: magnetic fields - stars: pulsars: general

\section{Introduction}

The influence of the magnetic field on the thermal history of neutron stars is twofold. On one hand, the field changes the character of heat transport providing some anisotropy to kinetic processes. Such anisotropic transport can result in a characteristic temperature difference between the poles and equator caused by largescale inhomogeneities of the magnetic field (see, e.g., Potekhin et al. 2003; Perez-Azorin et al. 2006). On the other hand, dissipation of the magnetic field provides an additional heating mechanism that can substantially alter the cooling evolution (Miralles et al. 1998). Even more or less standard for radiopulsars magnetic fields $\sim 10^{12}-10^{13} \mathrm{G}$ can maintain the surface temperature as high as $\sim 10^{5} \mathrm{~K}$ for $\sim 10-100 \mathrm{Myr}$ which is substantially higher than predicted by various cooling models. A general relativistic treatment of Joule heating in neutron stars undertaken by Page et al. (2000) led to results very similar to those of Miralles et al. (1998) since, as it was argued by Konar (2002), general relativistic corrections do not significantly influence the magnetic field decay in neutron stars.

The internal magnetic fields in neutron stars, however, can be substantially stronger than the "standard" surface field of radiopulsars, and they can produce much more efficient heating. The nature of pulsar magnetism is still the subject of debate. One of the possibilities is associated with the turbulent dynamo action that can amplify the magnetic field during the first $\sim 30-40 \mathrm{~s}$ of a neutron star's life when the star is subject hydrodynamic instabilities (Thompson \& Duncan 1993; Bonanno et al. 2003). Turbulent motion caused by instabilities in combination with rapid rotation that seems to be almost inevitable in protoneutron stars make a turbulent dynamo one of the most plausible mechanisms of pulsar magnetism. The characteristic feature of a turbulent dynamo in stars is that generation of the large-scale field is always accompanied by generation of small-scale fields that are approximately in equipartition with the turbulent motion. Therefore, if the field of pulsars is of dynamo origin, it should have a very complex geometry when dynamo action stops (see Urpin \& Gil 2004; Bonanno et al. 2005, 2006). Magnetic fields of various scales will be frozen into the crust that begins to form soon after the end of the unstable stage and then will decay due to the finite crustal resistivity.

Miralles et al. (1998) have considered the effect of Joule heating in old neutron stars with an age $t>10^{6} \mathrm{yrs}$, when the surface temperature is low and dissipation of even standard pulsar magnetic fields can change the thermal evolution substantially. In this paper, we treat the effect of dissipation during the early evolution of neutron stars $\left(t<10^{5}\right.$ yrs $)$, when neutron stars are much hotter. The crustal conductivity depends on the temperature and can be relatively low in hot neutron stars. Due to this, the field decay is rapid in young neutron stars with an age $\sim 10^{4}-10^{5}$ yrs. This type of behaviour has been well known since the paper by Urpin \& Muslimov (1992). The fast decay has been studied in detail by a number of authors (see, e.g., Urpin \& Konenkov 1997; Sengupta 1998; Mitra et al. 1999, etc.), and was rediscovered with no citation in recent calculations by Pons \& Geppert (2007). However, even such efficient field decay during the initial stage can influence the thermal evolution only if the magnetic field is sufficiently strong and, therefore, our study primarily addresses high magnetic field pulsars.

\section{The internal magnetic fields in pulsars}

The unstable stage lasts only $\sim 30-40 \mathrm{~s}$ in proto-neutron stars, but this is sufficient for dynamo action to generate a strong magnetic field because the turbulent motion is very fast. A turbulent 
dynamo can generate a large-scale magnetic field only if the star rotates rapidly and the rotation period $P_{0}$ is shorter than some "critical" value $P_{\text {cr }}(\sim 1 \mathrm{~s}$ in the simplest model). Most protoneutron stars likely rotate faster and, as a result, a dynamo should operate in these objects (Bonanno et al. 2003, 2005). The dynamo generates both the toroidal field that is essentially internal and vanishes at the surface and the poloidal field that is nonvanishing outside the star. The poloidal field is responsible for pulsar activity. Typically, the generated toroidal field is comparable to the poloidal one if the proto-neutron star rotates rigidly, but it can be substantially stronger if rotation is differential. For example, the toroidal field is $\sim 20-60$ times stronger than the poloidal one if rotation at the center is three times faster than that at the surface equator. The saturation field generated by the dynamo can be estimated by making use of the simplest model of $\alpha$-quenching (Bonanno et al. 2006). This yields for the generated large-scale field

$B_{0} \approx B_{\text {eq }} \sqrt{P_{\mathrm{cr}} / P_{0}-1}$

where $B_{\text {eq }}$ is the equipartition small-scale field, $B_{\text {eq }} \approx 4 \pi \rho v_{\mathrm{T}}^{2}$ where $v_{\mathrm{T}}$ is the turbulent velocity. The equipartition field varies during the unstable stage, rising rapidly soon after collapse and then decreasing when the temperature and lepton gradients are smoothed. At the peak, $B_{\mathrm{eq}} \sim 10^{16} \mathrm{G}$ in the convective zone (Thompson \& Duncan 1993) and $B_{\text {eq }} \sim(1-3) \times 10^{14} \mathrm{G}$ in the neutron-finger unstable zone (Urpin \& Gil 2004). However, $B_{\text {eq }}$ decreases and becomes smaller than the peak value as the protoneutron star cools down. The dynamo operates while the quasi-steady condition $\tau_{\text {cool }} \gg \tau$ is fulfilled with $\tau_{\text {cool }}$ being the cooling timescale and $\tau$ the turnover time of turbulence. The final strength of a generated small-scale magnetic field is approximately equal to $B_{\text {eq }}$ at the moment when the quasi-steady condition breaks down and $\tau \sim \tau_{\text {cool }}$. If $\tau \sim \tau_{\text {cool }}$, we have $v_{\mathrm{T}} \sim \pi \ell_{\mathrm{T}} / \tau_{\text {cool }}$ where $\ell_{\mathrm{T}}$ is the turbulent lengthscale. Then,

$B_{\text {eq }} \sim \sqrt{4 \pi \rho} v_{\mathrm{T}} \sim \pi \sqrt{4 \pi \rho} \ell_{\mathrm{T}} \tau_{\text {cool }}^{-1}$.

Estimate (2) yields $B_{\text {eq }} \sim(1-3) \times 10^{13} \mathrm{G}$ for the maximum possible lengthscale $\ell_{\mathrm{T}} \sim 1-3 \mathrm{~km}$ and $\tau_{\text {cool }} \sim$ few seconds.

Substituting estimate (2) into expression (1), we obtain

$B_{0} \approx \frac{\sqrt{4 \pi^{3} \rho} \ell_{\mathrm{T}}}{\tau_{\text {cool }}} \sqrt{\frac{P_{\mathrm{cr}}}{P_{0}}-1} \sim(1-3) \times 10^{13} \sqrt{\frac{P_{\mathrm{cr}}}{P_{0}}-1}$ G.

The shortest possible period is likely $\sim 1 \mathrm{~ms}$ and, hence, the strongest internal magnetic field generated by a dynamo can reach $\sim(0.3-1) \times 10^{15} \mathrm{G}$. The field at the surface is usually weaker than the internal field generated by a dynamo (Bonanno et al. 2006). Note that generation is more efficient in the outer layers because the parameter $\alpha$ that determines the efficiency of the dynamo is proportional to $\Omega$ and the density gradient (see, e.g., Rüdiger \& Kitchatinov 1993) but the latter is maximal in the outer layers.

If $P_{0}$ is longer than $P_{\mathrm{cr}}$, then the large-scale dynamo does not operate in proto-neutron stars but the small-scale dynamo is still efficient. One can expect that such neutron stars have only smallscale fields with the strength $B_{\text {eq }} \sim(1-3) \times 10^{13} \mathrm{G}$ and have no dipole field. Likely, slow rotation with $P_{0}>P_{\mathrm{cr}}$ is rather difficult to achieve in proto-neutron stars, and the number of such exotic objects is small.

It is difficult to predict the final disposition of the magnetic field after turbulent motion stops. The magnetic configuration generated by the dynamo can be unstable, for example, if it contains a strong toroidal component. MHD instabilities can lead to a rapid evolution on the Alfvén time scale and form a configuration with comparable poloidal and toroidal fields (see, e.g., Braithwaite \& Spruit 2006; Braithwaite \& Nordlund 2006). Therefore, it is unlikely that the toroidal field is substantially stronger than the poloidal one in neutron stars. When convection is exhausted, both large- and small-scale fields evolve under the influence of ohmic dissipation and buoyancy. Likely, formation of the crust provides the most important influence on the evolution of magnetic fields at this stage. Soon after convection stops, the neutron star cools down to the internal temperature $\sim(1-3) \times$ $10^{10} \mathrm{~K}$ at which neutrons and protons can form nuclei and clusters in matter with a density $\sim 10^{14} \mathrm{~g} / \mathrm{cm}^{3}$. When the temperature decreases progressively, nuclei can be formed at a lower density as well. Nuclear composition at high density is subject to many uncertainties and depends generally on the pre-history of the neutron star. For instance, the composition of the "ground state" matter (Negele \& Vautherin 1973) differs noticeably from that of the "accreted" matter (Haensel \& Zdunik 1990). The Coulomb interactions of nuclei leads to crystallization that occurs when the ion coupling parameter $\Gamma=Z^{2} e^{2} / a k_{\mathrm{B}} T$ reaches the critical value $\Gamma_{\mathrm{m}} \approx 170$ (Slattery et al. 1980$) ; a=\left(3 / 4 \pi n_{\mathrm{i}}\right)^{1 / 3}$ is the mean inter-ion distance, $n_{\mathrm{i}}$ and $Z$ are the number density and charge number of ions, respectively; $T$ is the temperature, and $k_{\mathrm{B}}$ is the Boltzmann constant. Then, the crystallization temperature is

$T_{\mathrm{m}} \approx 1.3 \times 10^{7} Z^{5 / 3} \mu_{\mathrm{e}}^{-1 / 3} \rho_{12}^{1 / 3} \mathrm{~K}$,

where $\mu_{\mathrm{e}}$ is the number of baryons per electron, and $\rho_{12}=$ $\rho / 10^{12} \mathrm{~g} / \mathrm{cm}^{3}$. For $\rho \sim 10^{13}-10^{14} \mathrm{~g} / \mathrm{cm}^{3}$, the crystallization temperature is $\sim 10^{10} \mathrm{~K}$. Therefore, the crust formation starts almost immediately after the end of the convective phase (or even before the end), and the magnetic field generated by the dynamo should be frozen into the crust.

\section{Joule heating in the crust}

Likely, the evolution of the magnetic field in the crust of hot neutron stars is mainly determined by the ohmic dissipation, and Joule heating has an important influence on the thermal evolution. The rate of Joule heating depends both on the geometry of the magnetic field and the conductive properties of the crust. In a strong magnetic field, the ohmic dissipation can be accompanied by Hall currents (Shalybkov \& Urpin 1997; Hollerbach \& Rüdiger 2002, 2004). These currents are nondissipative since they do not contribute directly to the rate of Joule heating. However, the Hall currents couple different modes and alter the magnetic configuration by redistributing the energy among modes. In this way, the Hall currents can indirectly affecr the rate of dissipation. Note also that, because of the non-linear nature of the Hall currents, they can generate magnetic features at the stellar surface with a smaller lengthscale than the background magnetic field. This has been known since the paper by Naito \& Kojima (1994) (see also Muslimov 1994; Shalybkov \& Urpin 1997) who argued that some neutron stars may have strong disordered magnetic fields at their surface due to the Hall effect. The same conclusion recently was reached by Pons \& Geppert (2007).

The influence of the Hall effect on the magnetic field is characterized by the Hall parameter, $\omega_{B} \tau$, where $\omega_{B}=\mathrm{e} B / m_{*} c$ is the gyrofrequency of electrons and $\tau$ is their relaxation time, $m_{*}$ is the electron effective mass. The Hall effect is unimportant if $\omega_{B} \tau \leq 1$, but it can influence the magnetic evolution if the field is sufficiently strong and $\omega_{B} \tau \gg 1$. Indeed, numerical 
simulations (Shalybkov \& Urpin 1997; Höllerbach \& Rüdiger $2002,2004)$ indicate that some acceleration of the decay can occur, particularly if the Hall parameter is very large, $\omega_{B} \tau>200$. This conclusion, however, turns out to be sensitive to the initial magnetic geometry. For a purely toroidal field, the Hall effect develops fine field structures, which cause the field to decay on the Hall time-scale rather than on a much longer ohmic timescale. However, the presence of even a relatively weak poloidal field ( $\sim$ few per cent) changes the result drastically, and dissipation proceeds approximately on the ohmic timescale. Since the magnetic field in the neutron star should have a rather complex geometry with both the poloidal and toroidal components, we can expect that departures in the rate of Joule heating caused by the Hall effect have to be small compared to the case when the ohmic dissipation is acting alone. Note that some simplified models describe the influence of the Hall effect on the magnetic evolution in terms of the decay on the Hall timescale (see, e.g., Cumming et al. 2004). From a comparison with numerical modelling, it is seen that this naive picture is insufficient. The Hall timescale often characterizes the period of oscillations rather than the decay timescale of magnetic modes. Such an oscillatory behaviour of the decaying magnetic modes was first obtained by Urpin \& Shalybkov (1997). Despite Pons \& Geppert (2007) having confirmed the earlier finding by Shalybkov \& Urpin (1997) and Höllerbach \& Rüdiger $(2002,2004)$ that the Hall effect does not substantially accelerate dissipation of realistic magnetic configurations, Aguilera et al. (2007) in their study of Joule heating in neutron stars assume confusingly that the Hall currents result in a fast decay of the magnetic field.

The field decay can also be influenced by the so-called Halldrift induced instability if it occurs in neutron stars (Geppert \& Rheinhardt 2002). These authors found that instability can occur which raises small-scale magnetic modes if the initial magnetic field is strong and its second spatial derivative is large enough. It is unclear, however, whether the effect of this instability on the dissipation rate is noticeable or not since a non-linear regime has not been considered. In particular, Hollerbach \& Rüdiger (2002) express doubts that a linear instability starting from small amplitude perturbations can be compatible with the cascade caused by the non-linear Hall effect. Since even this cascade leads only to small departures in the dissipation rate for realistic magnetic configurations (Shalybkov \& Urpin 1997; Höllerbach \& Rüdiger 2002, 2004), the effect of a linear instability should be very weak, if it exists. As far as the non-linear regime of instability is concerned, Geppert et al. (2003) assume that, at saturation, the amplitude of unstable perturbations can reach a significant fraction of the background field and, hence, the latter should decay on the Hall timescale. Since this obviously contradicts the observed lifetime of pulsars $\left(\sim 10^{8} \mathrm{yrs}\right)$, the authors assume that the Hall drift induced instability can operate only during part of the neutron star life whereas decay before and after such episodes is determined by the ohmic dissipation. According to Rheinhardt \& Geppert (2002), episodes occur when the age of a neutron star is $\geq 10^{5}$ yrs since the Hall parameter is not sufficiently large in younger stars. However, studies of pulsars based on methods of population synthesis (Bhattacharya et al. 1992; Hartman et al. 1997; Regimbau \& de Freitas Pacheco 2001; Faucher-Giguere \& Kaspi 2006) indicate that the magnetic field of radiopulsars decays little during their active lifetime. A significant magnetic field decay in radiopulsars likely can occur only in very young stars with age $\leq 10^{5}$ yrs since the number of these pulsars is small and they give a negligible contribution to any statistical analysis. Therefore, observations also do not support the idea of episodes of the Hall drift induced instability in neutron stars. Note that recent calculations of the non-linear field decay by Pons \& Geppert (2007) confirm the conclusion by Hollerbach \& Rüdiger (2002) that, even if the Hall-driven instability exists in neutron stars, it has no appreciable influence on the field decay.

\section{Statement of the problem}

To calculate the rate of Joule heating in young neutron stars, we consider a very simplified model. We assume that the magnetic field of neutron stars is generated by a turbulent dynamo at the beginning of their evolution. The dynamo $\alpha$-parameter is proportional to the density gradient and, hence, the dynamo is most efficient in the surface layers of proto-neutron stars (see Bonanno et al. 2005). Hence, one can expect that there exist electric currents in the outer crust just after the convective stage. The decay of currents is highly inhomogeneous since the electric resistivity depends on the density and varies by many orders of magnitude over the crust. The decay timescale is $t_{B} \approx 4 \pi \sigma L^{2} / c^{2}$, where $\sigma$ is the conductivity and $L$ is the lengthscale of the magnetic field, and currents deposited closer to the surface will decay faster because the conductivity is lower. Therefore, the thermal balance of young neutron stars with age $t \leq 10^{5}$ yrs can be influenced only by dissipation of currents located in the surface layers.

The decay of the crustal magnetic field is governed by the induction equation without the convective term

$\frac{\partial \boldsymbol{B}}{\partial t}=-\frac{c^{2}}{4 \pi} \nabla \times(\hat{R} \nabla \times \boldsymbol{B})$

where $\hat{R}=1 / \hat{\sigma}$ is the electric resistivity tensor and $\hat{\sigma}$ is the conductivity tensor. In a magnetized crust, components of the conductivity tensor are

$\sigma_{\|}=\sigma_{0}, \quad \sigma_{\perp}=\frac{\sigma_{0}}{1+\omega_{B}^{2} \tau^{2}}, \quad \sigma_{\mathrm{H}}=-\frac{\sigma_{0} \omega_{B} \tau}{1+\omega_{B}^{2} \tau^{2}}$,

where $\sigma_{\|}$and $\sigma_{\perp}$ are the components parallel and perpendicular to the magnetic field, $\sigma_{\mathrm{H}}$ is the so-called Hall component that determines the current perpendicular to both the magnetic and electric fields, $\sigma_{0}=e^{2} n_{\mathrm{e}} \tau / m_{*}$ is the coductivity at $\boldsymbol{B}=0, n_{\mathrm{e}}$ and $m_{*}$ are the number density of electrons and their effective mass, $\omega_{B}$ is the gyrofrequency of electrons, and $\tau$ is the relaxation time. If the magnetic field is sufficiently strong and $\omega_{B} \tau>1$, then the conductivity components across the magnetic field $\sigma_{\perp}$ and $\sigma_{\mathrm{H}}$ are suppressed, and only the conductivity along the magnetic field is essential. Components of the resistivity tensor $\hat{R}=1 / \hat{\sigma}$ are given by

$R_{\|}=R_{\perp}=1 / \sigma_{0}, \quad R_{\mathrm{H}}=\omega_{B} \tau / \sigma_{0}=B / e c n_{\mathrm{e}}$,

where $n_{\mathrm{e}}$ is the number density of electrons. In a strong magnetic field, the components parallel and perpendicular to the magnetic field are the same and are not suppressed by the magnetic field. The Hall component of resistivity $R_{\mathrm{H}}$ can be higher than $R_{\|}$if $\omega_{B} \tau>1$ that, generally, can be fulfilled in some layers of the neutron star.

We neglect the Hall term in our consideration and, strictly speaking, our results apply to neutron stars with not very strong magnetic fields. However, as was argued in previous studies (Urpin \& Shalybkov 1997; Höllerbach \& Rüdiger 2004; Pons \& Geppert 2007), even in a very strong magnetic field that can magnetize plasma, the Hall effect insignificantly changes the rate of dissipation if the magnetic field contains a poloidal component. The difference in the rate of dissipation between the model that takes into account the Hall effect and the hypothetical model 
that has the same initial magnetic configuration but evolves in accordance with Eq. (5) without the Hall effect does not exceed 10-20\%. This is much smaller than uncertainties in our knowlegde of the initial magnetic configuration or the chemical composition of the crust. Such accuracy is sufficient for our purposes, and we will mimic the field decay by Eq. (5).

We restrict our consideration to a dipolar field that can be described by the vector potential $\boldsymbol{A}=(0,0, S(r, t) \sin \theta / r)$, where $r$ and $\theta$ are the radius and polar angle, respectively. The function $S(r, t)$ obeys the equation

$\frac{\partial^{2} S}{\partial r^{2}}-\frac{2 S}{r^{2}}=\frac{4 \pi \sigma_{0}}{c^{2}} \frac{\partial S}{\partial t}$

with the boundary conditions $\partial S / \partial r+S / R=0$ at the stellar surface $r=R$ and $S \rightarrow 0$ in the deep crustal layers. The $\varphi$-component of the electric current maintaining the dipolar magnetic field is

$j_{\varphi}=-\frac{c}{4 \pi} \frac{\sin \theta}{r}\left(\frac{\partial^{2} S}{\partial r^{2}}-\frac{2 S}{r^{2}}\right)$.

We have for the rate of Joule heating $\dot{q}(r, \theta, t)=j_{\varphi}^{2} / \sigma_{0}$. For simplicity, nonsphericity is often neglected in cooling calculations. Therefore, we will use the polar-averaged expression for the rate of Joule heating. It is also convenient to normalize the function $S(r, t)$ to its initial value at the surface, $S(R, 0)$, which in turn can be related to the initial field strength at the magnetic equator $B_{\mathrm{e}}$ by $S(R, O)=R^{2} B_{\mathrm{e}}$. Then, the polar-averaged rate of Joule heating is given by

$\dot{q}=\frac{c^{2} R^{4} B_{\mathrm{e}}^{2}}{24 \pi^{2} r^{2} \sigma_{0}}\left(\frac{\partial^{2} s}{\partial r^{2}}-\frac{2 s}{r^{2}}\right)^{2}$.

In young neutron stars with age $t<10^{5} \mathrm{yrs}$, the conductivity of the outer crust and the outer part of the inner crust is determined by electron scattering on phonons. Scattering on impurities is important only in deep layers of the inner crust even if the impurity parameter is large (see, e.g., Jones 2004). We use the conductivity due to electron-phonon scattering calculated by Itoh et al. (1993). These conductivities depend on the temperature. Calculations presented here are based on the standard cooling scenario, which corresponds to a star with standard neutrino emissivities. We consider the field decay using the thermal history for the $1.4 M_{\odot}$ neutron star constructed with the equation of state of Friedman \& Pandharipande (1981) which is representative of intermediate equations of state. Our choice is compelled by the fact that models with the equation of state stiffer than that of Friedman \& Pandharipande agree better with data on the magnetic evolution of pulsars (Urpin \& Konenkov 1997).

Calculating the rate of Joule heating, we neglect its back influence on the thermal evolution of a neutron star. It is not the purpose of this paper to discuss in detail how the thermal and magnetic evolution are coupled. One can neglect this coupling if the rate of heating does not exceed the luminosity of a standard neutron star. Our approach allows us to obtain the lower limit on the field strength that can affect the thermal evolution due to Joule heating, and we compare this limit to observational data. Note, however, that even the temperature of magnetars which are believed to be heated by the decay of extremely strong fields $\geq 10^{14} \mathrm{G}$ is only a factor $\sim 2-3$ higher than the temperature of a standard cooling neutron star. Therefore, our calculations can provide an order of magnitude estimate for the rate of Joule heating in the case of such strong magnetic fields. This simplified approach can be useful in understanding the main qualitative features of Joule heating in magnetars. A study of the coupled early magnetothermal evolution of very strongly magnetized stars such as magnetars is a more complicated problem, and it will be considered in a forthcoming paper.

If the field in the surface layers is very strong, such as $B^{2} / 4 \pi>\mu$ where $\mu$ is the shear modulus of the crust, then the induction and momentum equations are coupled, and magnetic stresses can induce motion of the crustal material. Therefore, decay of the crustal magnetic field can change the Lorentz force and, generally, can lead to crustal motion. The shear modulus is $\mu \sim 0.1 n_{\mathrm{i}}(Z e)^{2} / a$ where $a=(3 / 4 \pi n)^{1 / 3}, Z$ and $n_{\mathrm{i}}$ is the charge and number density of ions (Strohmayer et al. 1991). The condition $B^{2} / 4 \pi>\mu$ is approximately equivalent to $\rho<$ $10^{9} B_{13}^{3 / 2} \mathrm{~g} / \mathrm{cm}^{3}$ where $B_{13}=B / 10^{13} \mathrm{G}$, and one should expect that Joule heating of the surface layers in high magnetic field pulsars is accompanied by motion in the crust. We will neglect, however, the contribution of this effect to heating.

\section{Numerical results}

In calculations, we assume that the magnetic field generated by a dynamo initially occupies the outer layer of the crust up to a density $\rho_{0}$ thus, initially, $B \neq 0$ at $\rho \leq \rho_{0}$. Since the field is confined to the crust, the density $\rho_{0}$ can be lower or equal to the density at the crust-core boundary, $\sim 2 \times 10^{14} \mathrm{~g} / \mathrm{cm}^{3}$. It is difficult to determine the inner boundary of the generation region from the physical data since both turbulent dynamo coefficients and stability properties of proto-neutron stars are subject to many uncertainties. Therefore, we perform calculations for a few different values of $\rho_{0}$. Note that the magnetic evolution is in good agreement with pulsar data if $\rho_{0} \sim 10^{13} \mathrm{~g} / \mathrm{cm}^{3}$ (Urpin $\&$ Konenkov 1997). This conclusion is in qualitative agreement with the dynamo models of neutron stars since the dynamo is efficient only in the surface layers where the density gradient is large. After the dynamo action stops in a proto-neutron star, the field diffuses into deeper layers and can be non-vanishing at $\rho>\rho_{0}$. The initial distribution of the magnetic field at $\rho<\rho_{0}$ is also uncertain but, fortunately, the main qualitative results are not crucially sensitive to this distribution. We consider two models of the initial distribution shown in Fig. 1. These models differ by the characteristic depth of currents with model 2 (shown in the dashed line) corresponding to a smaller depth than model 1 (solid line).

In Fig. 2, we plot the distribution of the rate of Joule heating, $\dot{q}$, for different ages. The distribution turns out to be complicated with a deep minimum that originates because, for a dipole field, the current density changes sign at some depth. The depth of this minimum increases with time due to diffusion of the magnetic field into deep layers. The origin of this minimum is caused by the general diffusive properties of the crustal magnetic field and can be understood from the simplest qualitative consideration. Indeed, if the initial distribution of $s$ is like those shown in Fig. 1, then the magnetic field will diffuse with time into deep layers with $\rho>\rho_{0}$ and, hence, the time derivative of $s$ will be positive somewhere at $\rho \geq \rho_{0}$, at least. On the another hand, the value of $s$ and the field strength at the surface will obviously decrease with time because of ohmic dissipation and,hence, we have $\partial s / \partial t<0$ at $r=R$. Since $s$ is a continuous function of the radius $r$ and $\partial s / \partial t$ has different signs at the surface and somewhere in deep layers, there always exists at least one point inside the neutron star crust where $\partial s / \partial t=0$. Combining Eqs. (8) and (9), we find that $j_{\varphi}=0$ at that point as well. Since $\dot{q} \propto j_{\varphi}^{2}$, the rate of heating is also vanishing at the same depth. In Fig. 2 , we do not show $\dot{q}$ at this depth in detail because the region where $\dot{q} \approx 0$ is very narrow. The first maximum corresponds to a relatively low density 


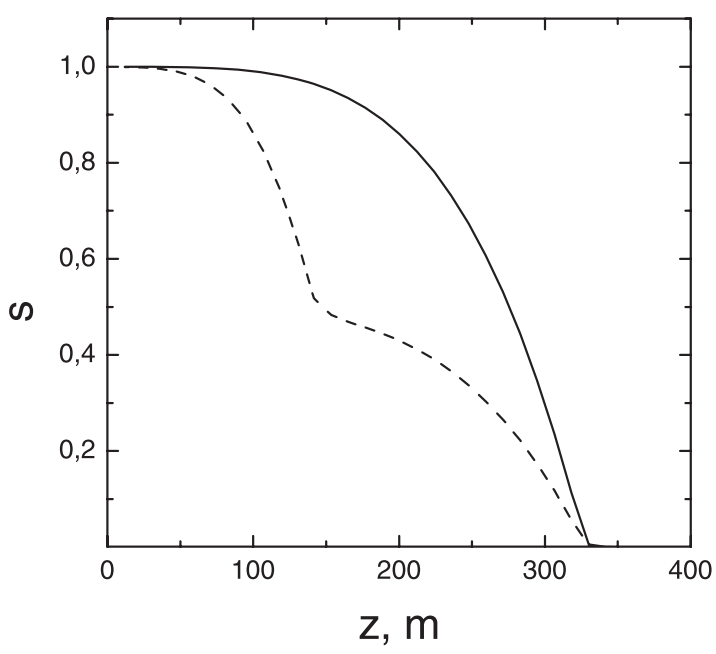

Fig. 1. The dependence of the initial distribution of $s(0, r)$ on the depth from the surface $z=R-r$ for the models 1 (solid line) and 2 (dashed line) and for $\rho_{0}=10^{13} \mathrm{~g} / \mathrm{cm}^{3}$ (the corresponding depth $z_{0}$ is approximately $330 \mathrm{~m}$.

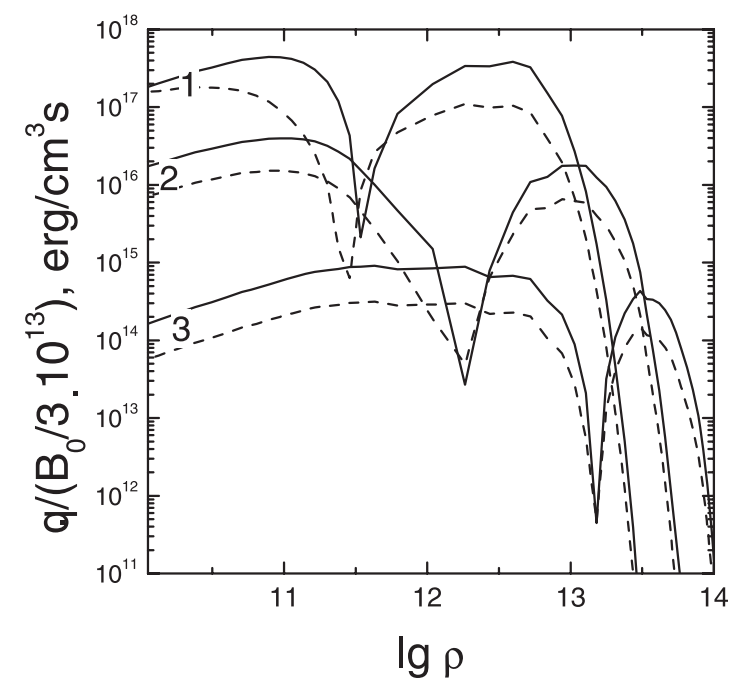

Fig. 2. The dependence $\dot{q}$ on $r$ for different ages and models. The solid and dashed lines corresponds to the models shown in the solid and dashed lines in Fig. 1. Curves $1-3$ show the distribution for $t=10^{2}$, $10^{3}$, and $10^{4} \mathrm{yrs}$, respectively.

$\sim 10^{10}-10^{12} \mathrm{~g} / \mathrm{cm}^{3}$, depending on the model. Initially, this maximum is located at a very low density, but it moves slowly inward with the time. After $10^{4} \mathrm{yrs}$, the maximum reaches a depth $\sim 300 \mathrm{~m}$ corresponding to $\rho \sim 10^{12} \mathrm{~g} / \mathrm{cm}^{3}$. At that time, the distribution of $\dot{q}$ is already rather flat in the surface layers. The second maximum of $\dot{q}$ is located substantially deeper, at densities $\sim 3 \times$ $10^{12}-3 \times 10^{13} \mathrm{~g} / \mathrm{cm}^{3}$, and it also moves inward with time. The position of the second maximum is less sensitive to the initial models because our models 1 and 2 differ mainly in the distribution of currents in the surface layers. Perhaps such a complicated distribution of $\dot{q}$ can result in a complicated radial dependence of the temperature in strongly magnetized stars. The rate of heating can reach a very high value $\sim 10^{16}-10^{18} \mathrm{erg} / \mathrm{cm}^{3} \mathrm{~s}$ or even higher if the initial magnetic field is stronger than $B \geq 3 \times 10^{13} \mathrm{G}$. The total rates of heating above and below the region where $\dot{q} \approx 0$ are of the same order of magnitude.

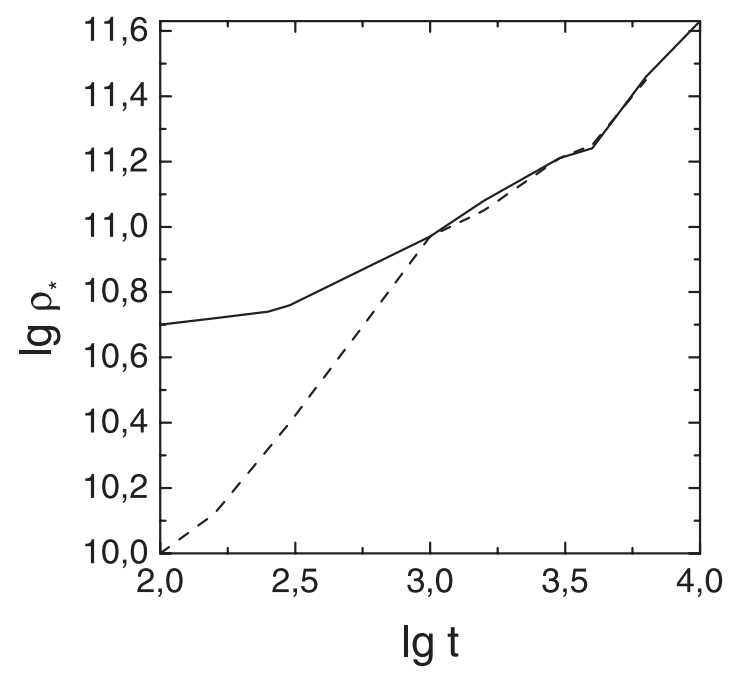

Fig. 3. The depth of the first maximum of $\dot{q}$ as a function of time for the models shown in Fig. 1.

In Fig. 3, we plot the evolution of the density $\rho_{*}$ where the rate of Joule heating is maximal for models 1 and 2 . This density corresponds to the first maximum of $\dot{q}$ (see Fig. 2). The location of the layer where the maxumum energy is released is important for understanding the energy budget in neutron stars (see, e.g., Kaminker et al. 2006). During the initial $\sim 10^{3}$ yrs, the depth of this layer is very small and corresponds to $\rho_{*}<10^{11} \mathrm{~g} / \mathrm{cm}^{3}$. At this stage, $\rho_{*}$ depends on the initial distribution of currents. Later on, at $t>10^{3}$ yrs, this depth becomes almost independent of the initial distribution. Up to $t \sim 10^{4}$ yrs, the maximum of Joule heating is located in the layers with a density lower than $4 \times$ $10^{11} \mathrm{~g} / \mathrm{cm}^{3}$. Likely, heat released in the layers with such a low density will be transfered to the surface and emitted by photons rather than diffusing to the core and being emitted by neutrinos.

In Fig. 4, we show the evolution of the total rate of Joule heating integrated over the crust volume, $\dot{Q}=\int \dot{q} \mathrm{~d} V$. For comparison, we also plot in this figure the photon luminosity of a neutron star with standard cooling. If the initial magnetic field is strong and the corresponding heating rate is above the dotted line, our results can provide only an order of magnitude estimate because Joule heating will influence the neutron star thermal evolution. The rate of Joule heating is calculated for three values of $\rho_{0}=10^{12}, 10^{13}$, and $10^{14} \mathrm{~g} / \mathrm{cm}^{3}$. For all these initial distributions of the magnetic field, the rate of Joule heating can reach rather high values $\sim 10^{34}-10^{35} \mathrm{erg} / \mathrm{s}$ during $\sim 10^{3} \mathrm{yrs}$ if the initial field strength is $3 \times 10^{13} \mathrm{G}$. This heating rate is higher than the luminosity of a standard neutron star without any additional heating mechanisms. At a given initial field strength, the heating rate is initially higher for smaller $\rho_{0}$, but it decreases faster with the time. For example, if the magnetic field initially occupies the surface layer with a density $\rho<\rho_{0}=10^{12} \mathrm{~g} / \mathrm{cm}^{3}$, then the heating rate at $t \sim 100 \mathrm{yrs}$ is approximately a factor of 10 higher than in the case of $\rho_{0}=10^{14} \mathrm{~g} / \mathrm{cm}^{3}$. However, the heating rate for $\rho_{0}=10^{12} \mathrm{~g} / \mathrm{cm}^{3}$ decreases very rapidly and becomes smaller already after $\sim 2 \times 10^{3}$ yrs. Generally, the rate of Joule heating can be high in young neutron stars. Even in pulsars with initial magnetic fields $\sim 3 \times 10^{13}$, Joule heating exceeds the photon luminosity during $\sim 10^{4}$ yrs if $\rho_{0} \sim 10^{13} \mathrm{~g} / \mathrm{cm}^{3}$. Therefore, young pulsars with such magnetic fields should be hotter than it is predicted by the standard cooling scenario. High magnetic field pulsars such as PSR J1847-0130 $\left(B \approx 9 \times 10^{13} \mathrm{G}\right)$ or PSR J1718$3718\left(B \approx 7 \times 10^{13}\right)$ can be in such a "heated" state even longer 


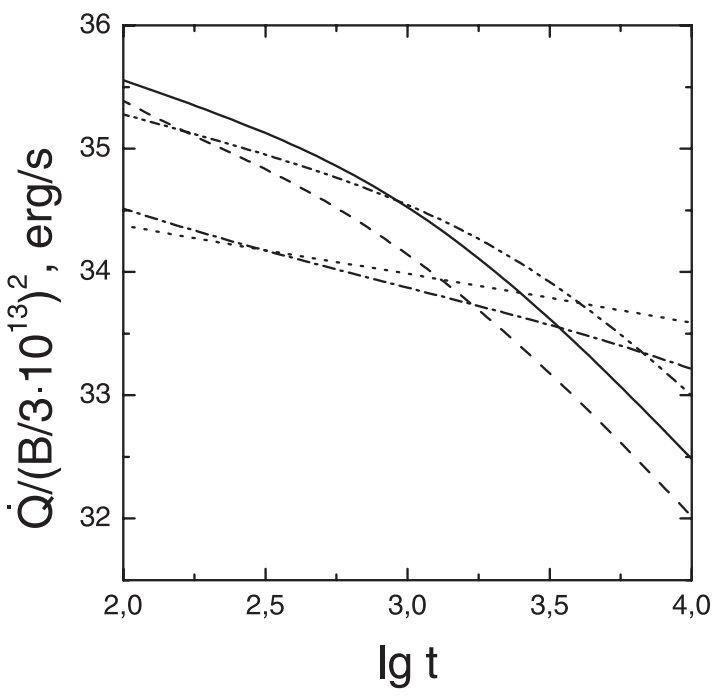

Fig. 4. The dependence of $\dot{Q}$ on time for different models. The magnetic field is normalized to $3 \times 10^{13} \mathrm{G}$. The solid and dashed lines correspond to the initial models 1 and 2 from Fig. 1, respectively, with $\rho_{0}=10^{12} \mathrm{~g} / \mathrm{cm}^{3}$. The dashed-and-dotted and dotted-and-dashed lines show $\dot{Q}$ for the model 1 with $\rho_{0}=10^{14}$ and $10^{13} \mathrm{~g} / \mathrm{cm}^{3}$, respectively. The dotted line shows the time dependence of the luminisity of a neutron star with no additional heating mechanism.

$\left(\sim 10^{5} \mathrm{yrs}\right)$. However, after that time, their thermal evolution will follow the standard scenario. Our calculations show that even the rate of heating required for magnetars, $\dot{Q} \sim 10^{35}-3 \times 10^{36} \mathrm{erg} / \mathrm{s}$, is reachable during the initial evolution if the initial magnetic field at the surface is $\sim 10^{14} \mathrm{G}$. Note that the rate of Joule heating depends not only on the field strength but also on the depth of the region where the magnetic field was generated. This dependence is rather sensitive and, generally, it is possible that the heating rate differs by more than an order of magnitude in pulsars of the same age and having approximately the same surface magnetic fields (for example, compare the solid and dash-anddotted lines in Fig. 4). Such different heating rates can lead to a substantial difference in the surface temperature in high magnetic field pulsars.

\section{Discussion}

We have considered dissipation of a magnetic field initially anchored to a neutron star crust. The magnetic field of neutron stars can be generated by a turbulent dynamo during a short hydrodynamically unstable phase at the beginning of their evolution. The mean-field dynamo is most effecient in the surface layers where the density gradient is large and, hence, the dynamo $\alpha$-parameter is maximal. Therefore, electric currents maintaining the magnetic configuration are likely located in the surface layers. Dissipation of electric currents is a highly inhomogeneous process because the density and, hence, conductivity varies by many orders of magnitude through the crust. The rate of dissipation decreases with increasing density and, hence, the field decay is slower in deep crustal layers. Dissipation of currents located in the surface layers proceeds on a relatively short time-scale and is accompanied by massive heat release. Joule heating is so efficient that it can even influence the thermal evolution of young neutron stars if the magnetic field is sufficiently strong.
The duration of the stage when Joule heating can affect the thermal evolution is determined by the strength of the magnetic field and the depth where this field is anchored. Both these quantities can vary over a wide range because they depend, for example, on poorly known details of the generation process and postcollapse hypercritical accretion that can essentially submerge the generated magnetic field (Chevalier 1989). At a given field strength, the rate of Joule heating is higher in a star where the field was initially anchored in the layer with a lower density. However, the time interval when this heating is important for the thermal evolution is shorter in such stars. On the contrary, if the field is anchored in a deeper layer, Joule heating is less effecient but it can contribute significantly in the thermal evolution over longer time.

Since the rate of Joule heating depends on two parameters it is generally possible that neutron stars with the same age and surface magnetic field can have substantially different heating rates and, hence, surface temperatures. This allows us to understand why the surface temperature of the high magnetic field pulsar PSR J1718-3718 is substantially lower than that of magnetars despite its magnetic field being approximately that of magnetars $\left(7.4 \times 10^{13}\right)$. The magnetic field of high magnetic field pulsars was likely anchored initially in layers with a lower density, and the stage of efficient Joule heating when their luminosity was comparable to that of magnetars is completed.

Our calculations show that additional heating provided by dissipation of the crustal magnetic field can even be comparable to the luminosity of magnetars $\left(\sim 10^{35}-10^{37} \mathrm{erg} / \mathrm{s}\right)$ if the magnetic field at the stellar surface is as high as $\sim(1-3) \times 10^{14} \mathrm{G}$. Generally, such a strong magnetic field can be generated in rapidly rotating proto-neutron stars by the turbulent $\alpha$-dynamo (Bonanno et al. 2006). Note that our calculations provide only the lower limit on the rate of Joule heating since we neglected the back influence of this heating on the crustal temperature. Additional heating will increase the temperature and decrease the conductivity and, hence, it will result in a higher dissipation rate if one consistently takes into account the coupling between the thermal evolution and field decay. For strongly magnetized stars with a surface field $\sim(1-3) \times 10^{14} \mathrm{G}$, the epoch when Joule heating is of the order of the magnetar luminosity can last as long as $\sim 10^{4}-10^{5}$ yrs if the magnetic field was initially anchored in the layers with $\rho_{0} \sim 10^{13} \mathrm{~g} / \mathrm{cm}^{3}$. This time is comparable to the estimated life-time of magnetars. If the initial field is weaker. $\sim 3 \times 10^{13} \mathrm{G}$, but is confined to the same layers, then the rate of Joule heating exceeds the standard luminosity of a cooling neutron star for a shorter time, $\sim 10^{3}$ yrs. During this time, the rate of heating is approximatelly one order of magnitude greater than the luminosity of a standard neutron star with the same age. Even in neutron stars born with a relatively weak magnetic field $\sim 3 \times 10^{12} \mathrm{G}$, Joule heating can influence the early thermal elolution but for a very short time and only if the magnetic field was initially confined to low density layers.

From our results, we conclude that many young neutron stars can ehxibit departures from the standard cooling scenario if the magnetic field is of crustal origin and if it is sufficiently strong. How large these departures are and how long Joule heating can manifest is determined by the initial strength of the magnetic field and its location. At the moment, observations only provide information on the surface temperature of neutron stars with an age greater than $\sim 1$ kyr. For stars with such age, our model predicts that departures from standard cooling can be expected only if the magnetic field is sufficiently strong, $B>(1-3) \times$ $10^{13} \mathrm{G}$. Therefore, recently discovered high magnetic field pulsars such as PSR J1847-0130 $\left(9.4 \times 10^{13} \mathrm{G}\right)$, PSR J1718-3718 


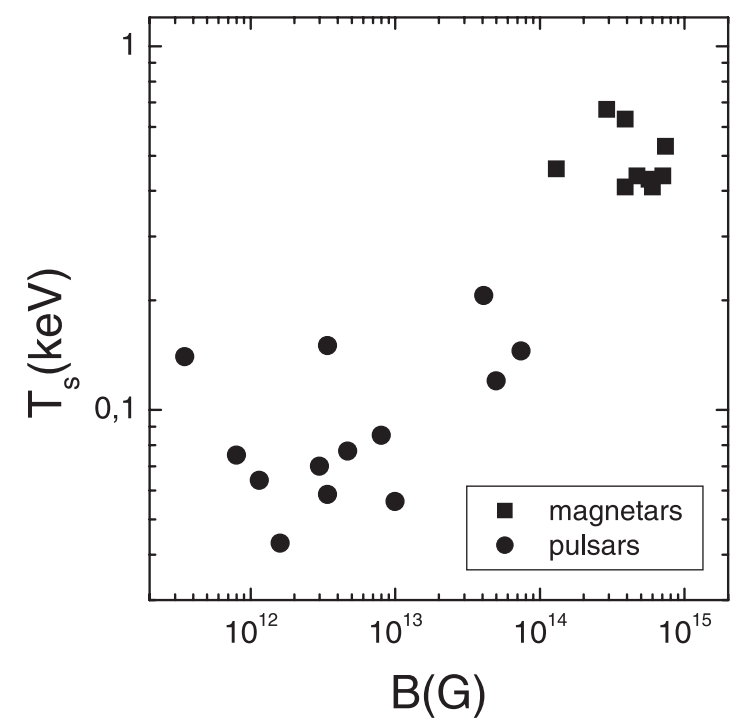

Fig. 5. The surface temperature $T_{\mathrm{s}}$ versus the magnetic field for 22 young neutron stars.

$\left(7.4 \times 10^{13} \mathrm{G}\right)$, PSR J1814-1744 $\left(5.5 \times 10^{13} \mathrm{G}\right)$, PSR J1119$6127\left(4.4 \times 10^{13} \mathrm{G}\right)$, and PSR B0154+61 $\left(2.1 \times 10^{13} \mathrm{G}\right)$ all can have a higher surface temperature than is predicted by standard cooling models without additional heating.

In Fig. 5, we plot the available data on the surface temperature $T_{\mathrm{s}}$ and magnetic fields $B$ of 22 young neutron stars. These data include 9 magnetars listed in Table 1 by Pons et al. (2007) which are likely young objects according to current theory. We also plot the data on $B$ and $T_{\mathrm{s}}$ for 13 radiopulsars with a spindown age shorter than $1 \mathrm{Myr}$. If the magnetic fields of these pulsars decay, their true age should be substantially less than the spin-down age and, likely, such pulsars are sufficiently young to be treated in our analysis. These 13 radiopulsars are listed in Table 1.

In accordance with our calculations, the thermal history of young pulsars with an age $\leq 10^{5}$ yrs can be influenced by Joule heating if the magnetic field at birth is stronger than $(1-3) \times$ $10^{13} \mathrm{G}$. Indeed, it seems that the surface temperature of high magnetic field pulsars with $B>10^{13} \mathrm{G}$ shows some trend to be higher than that of low magnetic field pulsars, in qualitative agreement with the prediction of our model. This difference cannot be accounted for by the influence of the magnetic field on the thermal conductivity, because the geometry and strength of the magnetic field are unimportant for the surface averaged thermal structure of neutron stars if the field strength is moderate, $B \leq 10^{14} \mathrm{G}$ (Potekhin et al. 2005). For such $B$, the magnetic field can affect the thermal evolution of neutron stars only via Joule heating, and the surface temperature should be proportional to approximately $B^{1 / 2}$. The data in Fig. 5 obviously are not sufficient to prove this dependence but, at least, they do not contradict it. More detections of the surface temperature in high magnetic field pulsars are needed, and they could provide strong evidence of the crustal origin of the magnetic field in neutron star.

It is important to consider coupled magnetothermal evolution by solving the heat and induction equations because characteristic timescales of the thermal and crustal magnetic evolution are approximately comparable in young neutron stars with $t<10^{5}$ yrs. However, this problem, taking into account the magnetically-induced anisotropy of the thermal conductivity and electric resistivity tensors, is far from being solved and the study
Table 1. The surface temperature and magnetic field of young radiopulsars.

\begin{tabular}{|c|c|c|c|}
\hline Source & $\begin{array}{l}k T \\
(\mathrm{keV})\end{array}$ & $\begin{array}{l}B \\
\left(10^{12} \mathrm{G}\right)\end{array}$ & Ref. \\
\hline RX J0822-4320 & $0.14-0.16$ & 3.4 & $\begin{array}{l}\text { Zavlin et al. (1999) } \\
\text { Pavlov et al. (1999) }\end{array}$ \\
\hline 1E 1207.4-5209 & $0.12-0.16$ & 0.35 & $\begin{array}{l}\text { Zavlin et al. (2004) } \\
\text { Gotthelf et al. (2007) }\end{array}$ \\
\hline Vela & $0.06-0.07$ & 3.4 & Page et al. (2004) \\
\hline PSR B1706-44 & 0.07 & 3.0 & Page et al. (2004) \\
\hline PSR J0538+2817 & 0.075 & 0.8 & $\begin{array}{l}\text { Levandowski et al. } \\
\text { (2004) }\end{array}$ \\
\hline Geminga & 0.043 & 1.6 & De Luca et al. (2005) \\
\hline PSR 1055-52 & 0.064 & 1.15 & De Luca et al. (2005) \\
\hline PSR B0656+14 & 0.077 & 4.7 & De Luca et al. (2005) \\
\hline PSR B2334+61 & 0.056 & 10 & $\begin{array}{l}\text { McGowan et al. } \\
\text { (2006) }\end{array}$ \\
\hline PSR J1718-3718 & 0.145 & 74 & Kaspi et al. (2005) \\
\hline PSR J1119-6127 & 0.206 & 41 & Gonzalez et al. (2005) \\
\hline CXOU J1819-1458 & 0.120 & 50 & Reynolds et al. (2006) \\
\hline PSR J1357-6429 & 0.085 & 8 & Zavlin (2007) \\
\hline
\end{tabular}

of particular problems of the magnetothermal evolution can help in understanding when Joule heating can affect neutron star cooling. From the result of the present study and conclusions obtained by Miralles et al. (1998), Joule heating can be important only in very young $\left(t<10^{4}-10^{5} \mathrm{yrs}\right)$ or in old $(t>10 \mathrm{Myr})$ neutron stars provided the magnetic field is sufficiently strong at birth. In stars with a moderate age, Joule heating seems to be inefficient.

Acknowledgements. This work has been supported by the Spanish Ministerio de Ciencia y Tecnologia grant AYA 2001-3490-C02. V.U. thanks Generalitat Valenciana and Universitat d'Alacant for a financial support and hospitality.

\section{References}

Aguilera, D., Pons, J., \& Miralles, J. A. 2008, ApJ, 673, L167

Bhattacharya, D., Wijers, R., Hartman, J., \& Verbunt, F. 1992, A\&A, 254, 198 Braithwaite, J., \& Nordlund, A. 2006, A\&A, 450, 1077

Braithwaite, J., \& Spruit, H. 2006, A\&A, 450, 1097

Bonanno, A., Rezzolla, L., \& Urpin, V. 2003, A\&A, 410, L33

Bonanno, A., Urpin, V., \& Belvedere, G. 2005, A\&A, 440, 199

Bonanno, A., Urpin, V., \& Belvedere, G. 2006, A\&A, 451, 1049

Chevalier, R. 1989, ApJ, 346, 847

Cumming, A., Arras, P., \& Zweibel, E. 2004, ApJ, 609, 999

De Luca, A., Caraveo, P., Mereghetti, S., Negroni, M., \& Bignami, G. 2005, ApJ, 623, 1051

Faucher-Giguere, C.-A., \& Kaspi, V. 2006, ApJ, 643, 332

Friedman, B., \& Pandharipande, V. 1981, Nucl. Phys. A, 361, 502

Geppert, U., \& Rheinhardt, M. 2002, A\&A, 392, 1015

Geppert, U., Rheinhardt, M., \& Gil, J. 2003, A\&A, 412, L33

Gonzalez, M., Kaspi, V., Camilo, F., Gaensler, B., \& Pivovaroff, M. 2005, ApJ, 630,489

Gotthelf, E., \& Halpern, J. 2007, ApJ, 664, L35

Haensel, P., \& Zdunik, J. 1990, A\&A, 227, 431

Hartman, J., Bhattacharya, D., Wijers, R., \& Verbunt, F. 1997, A\&A, 322, 477

Hollerbach, R., \& Rüdiger, G. 2002, MNRAS, 337, 216

Hollerbach, R., \& Rüdiger, G. 2004, MNRAS, 347, 1273

Itoh, N., Hayashi, H., \& Kohyama, Y. 1993, ApJ, 418, 405

Jones, P. B. 2004, Phys. Rev. Lett., 93, 1101

Kaminker, A., Yakovlev, D., Potekhin, A., et al. 2006, MNRAS, 371, 477

Kaspi, V., \& McLaughlin, M. 2005, ApJ, 618, L41

Konar, S. 2002, MNRAS, 333, 475 
Lewandowski, W., Wolszczan, A., Feiler, G., Konacki, M., \& Soltysinski, T. 2004, ApJ, 600, 905

McGowan, K., Zane, S., Cropper, M., Vestrand, W., \& Ho, C. 2006, ApJ, 639, 377

Miralles, J. A., Urpin, V., \& Konenkov, D. 1998, ApJ, 503, 368

Mitra, D., Konar, S., \& Bhattacharya, D. 1999, MNRAS, 307, 459

Muslimov, A. 1994, MNRAS, 267, 523

Naito, T., \& Kojima, Y. 1994, MNRAS, 266, 597

Negele, J., \& Vautherin, D. 1973, Nucl. Phys. A, 207, 298

Page, D., Geppert, U., \& Zannias, T. 2000, A\&A, 360, 1052

Page, D., Lattimer, J., Prakash, M., \& Steiner, A. 2004, ApJS, 155, 623

Pavlov, G., Zavlin, V., \& Truemper, J. 1999, ApJ, 511, 45

Perez-Azorin, J. F., Miralles, J. A., \& Pons, J. 2006, A\&A, 451, 1009

Pons, J., \& Geppert, U. 2007, A\&A, 470, 303

Pons, J., Link, B., Miralles, J., \& Geppert, U. 2007, Phys. Rev. Lett., 98, 1101

Potekhin, A., Yakovlev, D., Chabrier, G., \& Gnedin, O. 2003, ApJ, 594, 404

Potekhin, A., Urpin, V., \& Chabrier, G. 2005, A\&A, 443, 1025
Regimbau, T., \& de Freitas Pacheco, J. A. 2001, A\&A, 374, 182

Reynolds, S. P., Borkowski, K. J., Gaensler, B. M., et al. 2006, ApJ, 639, L71

Rheinhardt, M., \& Geppert, U. 2002, Phys. Rev. Lett., 88, 1103

Rüdiger, G., \& Kitchatinov, L. 1993, A\&A, 269, 581

Sengupta, S. 1998, ApJ, 501, 792

Shalybkov, D., \& Urpin, V. 1997, 321, 685

Slattery, V., Doolen, G., \& De Witt, H. 1980, Phys. Rev. A, 21, 2087

Strohmayer, T., Van Horn, H. M., Ogata, S., Iyetomi, H., \& Ichimaru, S. 1991, ApJ, 375, 679

Thompson, C., \& Duncan, R. 1993, ApJ, 408, 194

Urpin, V., \& Muslimov, A. 1992. MNRAS, 256, 261

Urpin, V., \& Konenkov, D. 1997, MNRAS, 292, 167

Urpin, V., \& Gil, J. 2004, A\&A, 415, 305

Zavlin, V. 2007, ApJ, 665, L143

Zavlin, V., Truemper, J., \& Pavlov, G. 1999, ApJ, 525, 959

Zavlin, V., Pavlov, G., \& Sanwal, D. 2004, ApJ, 606, 444 